\title{
Atom Scattering Picks Out the Heavyweights
}

\author{
Atomic-beam diffraction emerges as a viable approach to separating isotopes within the \\ beam.
}

by Holly Hedgeland*

$\mathrm{M}$ any great discoveries in science and technology have come from researchers who realized that a tangential finding of their experiment had potential for an entirely different purpose. Such serendipitous findings have brought us penicillin, superglue, microwave ovens, Viagra, and pacemakers-to name just a few. With the same ability to look beyond disciplinary silos, Kevin Nihill, Jacob Graham, and Steven Sibener at the University of Chicago, Illinois, have used diffraction of a beam of gaseous atoms-an approach traditionally employed to characterize the structure of surfaces-as a tool for separating the different isotopes within the beam gas itself [1].

Isotope separation is the process of concentrating particular isotopes of a chemical element by preferentially selecting them from a mixture of isotopes. The highest-profile example of isotope separation is the enrichment of uranium-235 from the heavier, more naturally abundant uranium-238. But isotope separation of other elements is critical to the provision of radioisotopes for medical applications, of "marker" atoms for following chemical reactions, and of ultrapure silicon for the semiconductor industry.

Various experimental approaches to isotope separation have been used, from those of the Manhattan Project based on the methods of gaseous diffusion and mass spectrometry, to later techniques involving distillation and centrifugation. Chemical methods, such as the so-called Girdler sulphide process for filtering heavy water out of natural water [2], have also been commercially viable, although they are very specific in their application. A common feature of these approaches is the need to pass the isotopic mixture through multiple iterations of the separation process. This "cascade" technique is necessary to achieve the required level of isotopic purity, but it is energy intensive.

More recent developments have used tunable lasers to ionize only the desired isotope, which is then collected by

\footnotetext{
*School of Physical Sciences, The Open University, Walton Hall, Milton Keynes MK7 6AA, United Kingdom
}

electrostatic deflection. The separation here can be much more efficient than the earlier approaches, but because of other technical challenges, the method has met with only limited application within the nuclear-technology industry [3]. Medical and technological applications requiring isotope separation motivate the search for new and more efficient approaches that are viable outside the nuclear niche.

The Chicago group's approach has the potential to fill this gap. The experiment fires a fast and well-collimated beam of neon gas-containing the isotopes neon-22 $\left({ }^{22} \mathrm{Ne}\right)$ and the more naturally abundant neon-20 $\left({ }^{20} \mathrm{Ne}\right)$ - at a highly reflective target of methyl-coated silicon (Fig. 1). The periodic structure of the silicon surface lattice acts as a diffraction grating to the low-energy beam of neon atoms, with the two different mass isotopes scattered to slightly different angles, as defined by Bragg's law. This angular separation enables the collection of the desired ${ }^{22} \mathrm{Ne}$ beam. By analyzing the composition of this beam, the team found that it was 3.5 times more enriched with ${ }^{22} \mathrm{Ne}$ than the original beam. In addition, the group showed that the isotope selection could be improved further in the future through an additional temporal effect.

To understand this temporal effect, we need to look at the physics of the neon beam and of the diffraction more closely. The beam is formed by expanding neon gas through a cooled nozzle with a fine, micrometer-sized aperture into a vacuum, resulting in a well-collimated beam of atoms moving at supersonic speeds. It is crucial to this experiment that the collimation of the beam produce a narrow spot of high intensity on the silicon surface and also that the incident beam atoms have the narrow velocity distribution associated with a supersonic beam [4]. As the temperature and energy of the beam atoms are constant, the difference in the masses of the isotopes will result in a small difference in their mean velocities and effective mean wavelengths. It is the difference in wavelength that yields the spatial separation of the diffracted beams demonstrated here. The difference in the mean velocities is, on the other hand, linked to the temporal effect that could improve the isotope selection further. The authors show that this improvement could be achieved by positioning a "chopper," a rotating disk with slots in it, in the beam path to break the incoming beam into sep- 


\section{Phys̄ị̂s}

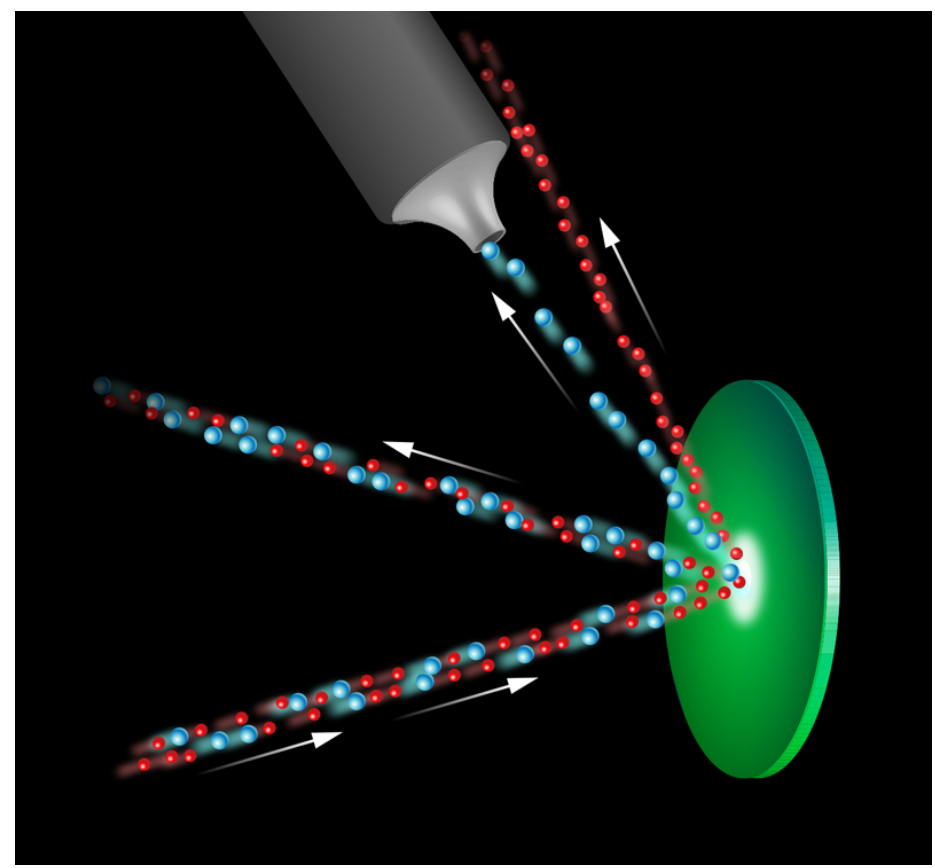

Figure 1: Nihill, Graham, and Sibener [1] have demonstrated that atomic-beam diffraction could be used as a method of isotope separation. The team used a methyl-coated silicon target to separate a beam composed of two isotopes of neon (shown in red and blue). Apart from the directly reflected beam, a fraction of each of the two isotopes making up the beam scatters to slightly different angles after hitting the target, giving the potential to collect the desired, but naturally less abundant, isotope separately. (APS/Alan Stonebraker)

arated pulses. As the velocities of the two isotopes vary slightly, by the time the beam has scattered from the target and reached the collector, the pulse of the desired isotope could be temporally separated. Placing a second, appropriately synchronized chopper within the collection system to pass only the desired pulse would increase the enrichment factor by reducing the amount of the unwanted isotope that is accidentally collected.

The Chicago team's work is an elegant proof of principle, and it is not hard to see how their approach could be used to make a practical isotope-selection apparatus, with the potential for the gas collected to be further purified for the desired isotope by being recycled through the process. Compact beam sources with narrow velocity distributions are an established technology [5, 6], and recycling systems have already been applied when costly helium-3 has been used as the beam gas [7]. It is also clear that the yield of the system would be improved if it were possible to collect multiple diffracted beams; this is a challenge already being tackled in the context of helium-atom microscopy [8]. In addition, altering the target from the methyl-coated silicon used here gives the potential to adjust the spatial separation of the diffracted beams as the surface lattice parameter changes, while maintaining a high surface reflectivity. A variation on this level is likely to be a prerequisite for handling different atomic species. However, the development of reflective elements for atom beams is a well-placed complementary research area [9].

In moving to a wider range of atomic species, the approach will face technical challenges if clustering of the atomic species within the beam becomes likely or if a seed gas is required for heavier atoms [4, 10]. However, turning the principle demonstrated here by the Chicago group into a working prototype of a new isotope-separation apparatus requires no significant change in instrumentation technology, and it promises to result in an energy-efficient and comparatively low-cost approach to separating isotopes.

This research is published in Physical Review Letters.

\section{REFERENCES}

[1] K. J. Nihill, J. D. Graham, and S. J. Sibener, "Separation of Isotopes in Space and Time by Gas-Surface Atomic Diffraction," Phys. Rev. Lett. 119, 176001 (2017).

[2] G. Neuneck, "The Atomic Bomb Reveals the Political Responsibility of Science," in Time, Quantum and Information, edited by L. Castell and O. Ischebeck (Springer, Berlin, 2004).

[3] F. Slakey and L. R. Cohen, "Stop Laser Uranium Enrichment," Nature 464, 32 (2010).

[4] G. Scoles, Atomic and Molecular Beam Methods Volume I (Oxford University Press, New York, 1998).

[5] M. Barr, K. M. O'Donnell, A. Fahy, W. Allison, and P. C. Dastoor, "A Desktop Supersonic Free-Jet Beam Source for a Scanning Helium Microscope (SHeM)," Meas. Sci. Technol. 23, 105901 (2012).

[6] B. A. J. Lechner, H. Hedgeland, W. Allison, J. Ellis, and A. P. Jardine, "Note: A New Design for a Low-Temperature HighIntensity Helium Beam Source," Rev. Sci. Instrum. 84, 026105 (2013).

[7] H. Hedgeland, P. R. Kole, W. Allison, J. Ellis, and A. P. Jardine, "An Improved High Intensity Recycling Helium-3 Beam Source," Rev. Sci. Instrum. 80, 076110 (2009).

[8] A. Fahy, M. Barr, J. Martens, and P. C. Dastoor, "A Highly Contrasting Scanning Helium Microscope," Rev. Sci. Instrum. 86, 023704 (2015).

[9] G. Anemone, A. Al Taleb, S. D. Eder, B. Holst, and D. Farías, "Flexible Thin Metal Crystals as Focusing Mirrors for Neutral Atomic Beams," Phys. Rev. B 95, 205428 (2017).

[10] O. F. Hagena and W. Obert, "Cluster Formation in Expanding Supersonic Jets: Effect of Pressure, Temperature, Nozzle Size, and Test Gas," J. Chem. Phys. 56, 1793 (1972).

10.1103/Physics.10.116 